\section{Advanced IC \\ Packaging Center in I-Shou University}

\section{E-mail: hchsu@isu.edu.tw}

I-Shou University (ISU) was established in 1990 by Chairman Lin, I-Shou. At present (2013), ISU has 41 departments, 18 postgraduate programs, and 7 doctorate programs. The number of students exceeds 16,000 and keeps growing. Advanced IC Packaging Center (AICP) was launched in 1994 by Dr. Fu, Shen-Li, Chair Professor and first president of ISU. Through research, consultancy and talent training programs, together with government grants and industry funds, AICP becomes an industry-oriented research center of IC packaging technology in southern Taiwan. The goal of AICP is to research and develop of new materials, processes and designs in the packaging technology of advanced electronic products.

There are four main research groups by function in AICP, namely electrical, material, thermal and stress. Professors from Electronic Department, such as Shen-Li Fu, Meiso Yokoyama, Yu-Jung Huang, Lih-Shan Chen and Shui-Hsiang Su are in charge of electrical and electronics fields. Professor Horng-Jer Tai from Chemical Engineering and Professor Cho-Liang (Mark) Chung from Materials Science and Engineering are related to material researches. Professor Hsiang-Chen Hsu and Li-Ming Chu from Mechanical Department are involved in thermal and stress studies, respectively.

AICP's achievements in various aspects including IP produced, awards obtained and technology breakthrough made. Related researches are: low-temperature co-fired ceramic (LTCC) multilayer substrate, high-density interconnected inner via hole (HDIIVH) substrate, organic light-emitting diode (OLED), polymeric substrate for flexible carbon nanotube displays, photodetecting system on flexible organic substrate $\cdots$ etc.

AICP has fabricated pentacene-based organic thin-film transistors (OTFTs) on a flexible substrate using a UV-patternable polymer material as the gate insulator. In addition to its solutionprocess able capability, the gate insulator is directly patterned by UV light in a low temperature process. The effect of UV/ozone post-treatment on the polymer dielectric is confirmed using a Fourier transform infrared (FT-IR) spectrometer. Owing to its highly desired characteristics such as photo patternability and lowtemperature process, the polymer dielectric is feasible for lowcost, large-area flexible device applications.

The materials group has developed highly flexible, durable and transparent poly(3,4-ethylenedioxythiophene)-based conducting dispersions with the incorporation of rubbery latexes, which can be spin-coated or cast onto various substrate. It can be used in a roll-to-roll process for flexible electronics. Among other achievements, AICP has synthesized an intelligent temperature sensitive material by combining thermally responsive poly(Nisopropyl acrylamide)-based copolymers with a conductive carbon black. It has also synthesized various polymers which can be employed to fabricate molding compounds for electronic encapsulation, and has developed various techniques to characterize the compounds.

Thermal design and reliability analysis on thermo-hygro-vapor pressure coupled effects on the IC packaging are established. Fickian and non-Fickian law of transient diffusion are solved by using finite element analysis to evaluate the overall moisture distributions and predict the thermal-induced strain, hygroscopic swelling deformation, and thermo-hygro-vapor pressuremechanical stress distributions. As the characteristic size of the thin film of Intermetallic Compound (IMC) reduces to nanoscale, finite element method is not capable to investigate the strain/ stress behavior on the nanostructure. A coupled molecular dynamics (MD) simulation with finite element approach is developed. Nano mechanics behaviors can be described by MD simulation while finite element method is applied to study meso continuum behaviors.

The recent 3D IC stacking technology, chip-to-chip circuit design suitable for three-dimensional integrated circuit stacked applications is also implemented in AICP. The proximity data link is performed via capacitive couplings between the top metals of face-to-face dies for three-dimensional system integration. The proposed AC coupled chip-to-chip circuit was simulated using HSPICE with TSMC $1.8-\mathrm{V} 0.18-\mu \mathrm{m}$ CMOS process $1 \mathrm{P} 6 \mathrm{M}$ technology file. The proposed circuit is not only with the self-test characteristics but also suitable for high-speed interconnection applications with differential signal transmission up to $2.5 \mathrm{Gbps}$.

Based on the above performances, AICP is eager to exploring the industrial-academia collaboration and continually paying attention to the latest trends of global micro-system, packaging and circuit technology. AICP welcome IC packaging experts all over the world to visit and sharing experience.

(Hsiang-Chen Hsu, Ph. D./I-Shou University)

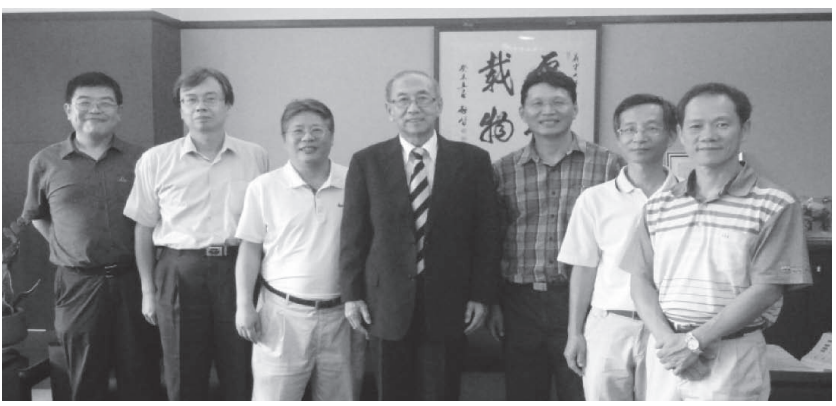

Group photo of AICP in ISU, from left, Professors: Hsiang-Chen Hsu, Lih-Shan Chen, Yu-Jung Huang, Shen-Li Fu (suited), Cho-Liang Chung, Horng-Je Tai and Shui-Hsiang Su.

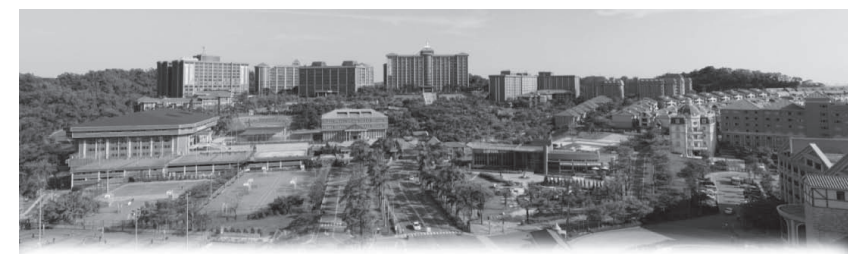

Main campus of I-Shou University, Kaohsiung City, Taiwan, ROC 